\title{
High storage capacity of synfire chains in large- scale cortical networks of conductance-based spiking neurons
}

\author{
Chris Trengove ${ }^{1 *}$, Cees Van Leeuwen², Markus Diesmann ${ }^{1,3}$ \\ From Nineteenth Annual Computational Neuroscience Meeting: CNS*2010 \\ San Antonio, TX, USA. 24-30 July 2010
}

We demonstrate stable dynamics of synchronous pulse packet propagation and global asynchronous irregular (AI) activity in a sparse network of $10^{5}$ neurons in which excitatory connectivity is derived from a random superposition of synfire chains having a total length of up to 80,000 pools. This is the largest amount of synfire chains to have been stably embedded in a network model of spiking neurons: about two orders of magnitude great than achieved previously [1-3]. Key features of the model which allow this high storage capacity are: (1) conductance-based rather than current-based synapses; and (2) inhibitory neurons in the synfire chain pools. As noted recently [3] the use of conductance over current based results in a narrower membrane potential distribution with a mean closer to threshold, which is more favourable for pulse-packet propagation at realistic levels of AI activity and background input.

Recurrent dynamics of the AI state is driven by the propagating pulse packets which deliver random excitatory and inhibitory 'background input' to the rest of the network. The equilibrium dynamical behaviour of the network depends on a critical level of excitatory and inhibitory background input above which synfire wave propagation ceases to be viable. In our simulation protocol synfire waves are initiated by ongoing, intermittent delivery of external input pulses. In the stable regime, an upper limit in the number of simultaneously propagating pulse packets is reached, typically $\sim 5$-30 waves, above which further waves are only successfully initiated at the expense of existing waves.

Using a semi-analytical framework, storage capacity, and the number of pulse packets that can propagate

\footnotetext{
* Correspondence: ctrengove@brain.riken.jp

${ }^{1}$ Brain and Neural Systems Team, RIKEN, Wako City, Saitama, 351-0198, Japan
}

simultaneously, is calculated. Mean field analysis is used to estimate the stability and spiking rate of the AI state, combined with a numerical determination of the stability of wave propagation. Results agree qualitatively with simulations and correctly predict the order of magnitude of storage capacity. Departures from mean field theory are observed, most notably the greater fluctuations in the AI state particularly as the upper limit of storage is approached, which impacts upon the stability of pulse packet propagation and reduces the maximum number of simultaneously propagating waves.

We also demonstrate in a modified version of this architecture that includes branching chains how synfire waves can be generated and maintained endogenously, using the same mechanism which stabilises the number of synfire waves at upper limit determined by the critical level of background input.

\section{Author details}

'Brain and Neural Systems Team, RIKEN, Wako City, Saitama, 351-0198, Japan. 2Dynamics Laboratory, RIKEN, Wako City, Saitama, 351-0198, Japan. ${ }^{3}$ Research Unit, Brain Sciences Institute, RIKEN, Wako City, Saitama, 351-0198, Japan.

\section{Published: 20 July 2010}

\section{References}

1. Aviel $Y$, Horn D, Abeles M: Memory capacity of balanced networks. Neural Comput 17:691-713.

2. Schrader S, Grun S, Diesmann M, Gerstein G: Detecting synfire chain activity using massively parallel spike train recording. J Neurophysiol 100:2165-2176.

3. Kumar A, Rotter S, Aertsen A: Conditions for propagating synchronous spiking and asynchronous firing rates in a cortical network model. J. Neurosci 28:5268-5280.

\section{doi:10.1186/1471-2202-11-S1-F1}

Cite this article as: Trengove et al:: High storage capacity of synfire chains in large-scale cortical networks of conductance-based spiking neurons. BMC Neuroscience 2010 11(Suppl 1):F1. 